\title{
Floating and sinking of a pair of spheres at a liquid-fluid interface
}

\author{
Himantha Cooray, ${ }^{\dagger, \ddagger}$ Pietro Cicuta, ${ }^{\dagger}$ and Dominic Vella*, \\ $\dagger$ Cavendish Laboratory, University of Cambridge, J.J. Thomson Avenue, Cambridge CB3 \\ OHE, U.K. \\ $\ddagger$ Institute of Theoretical Geophysics, Department of Applied Mathematics and Theoretical \\ Physics, University of Cambridge, Wilberforce Road, Cambridge CB3 0WA, U. K. \\ \Mathematical Institute, University of Oxford, Woodstock Rd, Oxford OX2 6GG, U. K. \\ E-mail: dominic.vella@maths.ox.ac.uk
}

\begin{abstract}
Spheres floating at liquid-fluid interfaces cause interfacial deformations such that their weight is balanced by the resultant forces of surface tension and hydrostatic pressure, whilst also satisfying a contact angle condition. Determining the meniscus shape around several floating spheres is a complicated problem because the vertical locations of the spheres and the horizontal projections of the three-phase contact lines are not known a priori. Here, a new computational algorithm is developed to simultaneously satisfy the nonlinear Laplace-Young equation for the meniscus shape, the vertical force balance, and the geometric properties of the spheres. We implement this algorithm to find the interface shape around a pair of floating spheres and the horizontal force required to keep them at a fixed centre-centre separation. Our numerical simulations show that the ability of a pair of spheres to float (rather than sink) depends on their separation. Similar to previous work on horizontal cylinders, sinking may be induced at close range for small spheres that float when isolated. However, we also discover a new
\end{abstract}


and unexpected behavior: at intermediate inter-particle distances, spheres that would sink in isolation can float as a pair. This effect is more pronounced for spheres of radius comparable to the capillary length, suggesting that this effect is a result of hydrostatic pressure, rather than surface tension. An approximate solution confirms these phenomena and shows that the mechanism is indeed the increased supporting force provided by hydrostatic pressure. Finally, the horizontal force of capillary attraction between the spheres is calculated using the results of the numerical simulations. These results show that the classic linear superposition approximation (due to Nicolson) can lose its validity for relatively heavy particles at close range.

\section{Introduction}

The liquid meniscus that forms around a vertical object piercing a liquid-fluid interface is familiar from everyday life and is perhaps the most common demonstration of interfacial tension. The shape of such a meniscus is well-known to be governed by the Laplace-Young equation, ${ }^{1}$ which balances the hydrostatic pressure within the liquid and the pressure jump across the interface due to surface tension. The solution of the Laplace-Young equation with boundary conditions corresponding to a planar wall piercing the surface may be found analytically and is given in many introductory textbooks on fluid mechanics. ${ }^{2}$ In other geometries, asymptotic techniques allow approximate solutions to be found for the meniscus around objects of constant cross section; for example, the meniscus around a vertical cylinder has been calculated asymptotically, in the limit of very small cylinder radius. ${ }^{3}$ These classic techniques are useful in applications for which the shape of the contact line takes a simple geometrical form that is known beforehand. However, more often applications involve floating objects for which the position and shape of the contact line must be determined as part of the solution to the problem. These applications include a range of self-assembly scenarios $^{4-7}$ while various insects that live on the surface of water and deform the liquid meniscus noticeably. ${ }^{8,9}$ This paper considers the meniscus around two rigid, floating spheres. 
For a single sphere, the contact line remains circular although the position at which the sphere floats is determined by the balance between the restoring forces (provided by surface tension and buoyancy) and the sphere's weight. The uncertainty in the vertical position of the sphere means that the radius of the contact line is not known a priori. Nevertheless, a relatively simple modification of the calculation for a vertical cylinder allows one to determine the level at which the sphere floats, the radius of the contact line and the shape of the meniscus around it. ${ }^{10-12}$ For the case of several spheres, however, the situation is considerably more complicated. The presence of one or more additional spheres nearby causes the contact line to tilt; it is no longer circular and the meniscus loses its axisymmetry. Again, the requirement that the vertical forces (sphere weight as well as the forces from surface tension and buoyancy) balance means that the vertical position of the contact line is not known and now even the shape of the contact line is not known.

An obvious consequence of a tilted contact line is that the horizontal forces acting on a sphere in the presence of other spheres do not, in general, balance. This gives rise to a horizontal force that causes identical spheres to clump together - an effect that can be observed in a bowl of breakfast cereal ${ }^{13}$ and is used by water-walking insects to climb the surface of ponds. ${ }^{14}$ The horizontal capillary force can also be used in combination with magnetic forces to tune self-assembly, and even an artificial swimmer. ${ }^{15,16}$ The calculation of this flotation force is usually performed approximately by assuming that the menisci of individual spheres may be linearly superposed; the force is then assumed to arise from the gravitational potential energy that each sphere has as a result of being displaced by the menisci of the other spheres - an approximation often named for Nicolson. ${ }^{11,13,17}$ Variations on this approach have yielded similar results. ${ }^{18,19}$ This approximation has been validated by comparison with the results of numerical computations for the case of two infinitely long cylinders lying horizontally at an interface ${ }^{11,20}$ but has only recently been shown to be the mathematically correct approach in the limit of vanishing cylinder radius. ${ }^{21}$ The analogous approach for vertical cylinders piercing an interface has also been validated numerically ${ }^{22}$ 
but we are not aware of a direct comparison between numerical and approximate results for the case of floating spheres. In this paper, we present numerical results that provide a direct comparison with the usual approximate methods based on the Nicolson Approximation, as well as the first direct test of its validity.

A less obvious consequence of the contact line tilting caused by the presence of other floating objects is the associated modification of the vertical supporting force supplied by surface tension and hence the vertical force balance. The analogous problem has been studied for two cylinders lying horizontally at an interface ${ }^{23}$ where it was shown that, under certain conditions on the density and size of the objects, the cylinders may float when well-separated but sink if they become too close. When large numbers of dense objects float in contact with one another, they form a 'capillary raft' that is supported by a combination of hydrostatic and capillary forces. Previous studies have shown that such rafts float, provided that their lateral size remains below a critical threshold that depends on their density in both the two-dimensional ${ }^{23}$ and axisymmetric cases. ${ }^{24}$ Several other experiments have been carried out to determine the collective floating behavior of many particles: the loads supported by rough copper granules aggregated on water have been measured,${ }^{25}$ and the loading capacity of structures produced by aggregation of superhydrophobic aluminium hexagons have been studied experimentally. ${ }^{26}$ However, the problem of how small numbers of spheres affect each other's force balance has not been considered previously with attention focussing instead on the maximum density a single object can have and still float. ${ }^{10,12,27-29}$

This paper presents a precise numerical solution for the floating and sinking behavior of a system consisting of a pair of identical spheres. We show that, even in this simple system, the collective behavior of floating spheres is qualitatively different to that of isolated spheres. We begin by considering the well known case of an isolated sphere so that the relevant theoretical results are readily at hand. We then go on to develop the mathematical problem for the flotation of two spheres and present an algorithm to solve this problem, together with the numerical results of this algorithm. We also present an approximate 

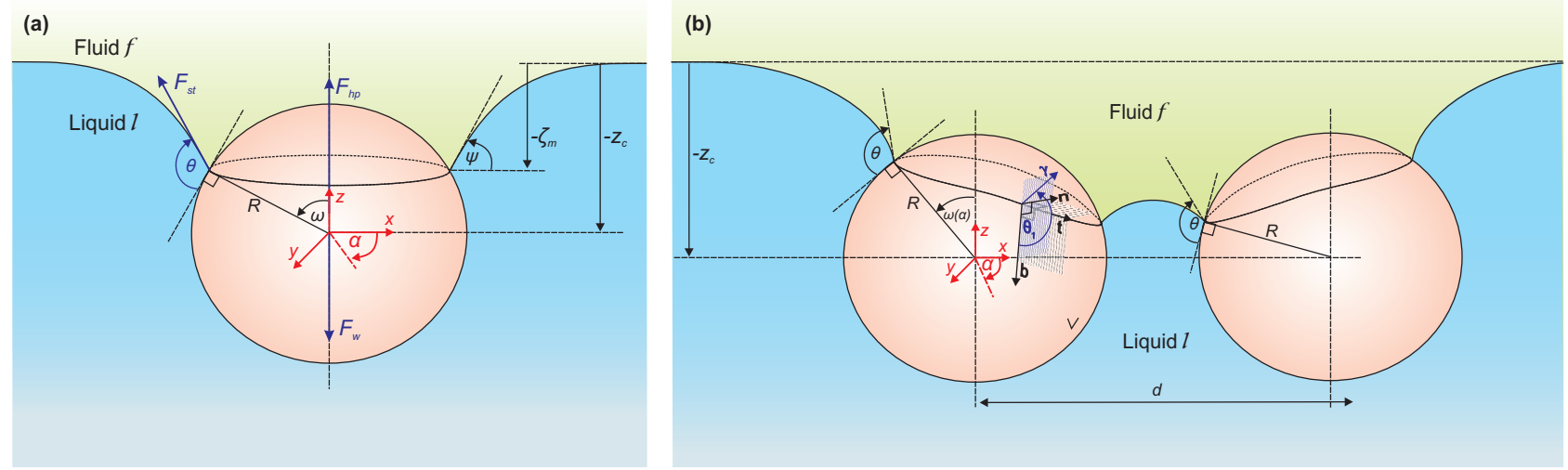

Figure 1: (a) Schematic diagram showing the typical configuration of an isolated sphere floating at a liquid-fluid interface. Here $R$ denotes the radius of the sphere and $\theta$ is the contact angle, which gives the boundary conditions where the interface meets the sphere. Far away, the interface is required to return to the unperturbed liquid level, indicated by the horizontal dashed line. $\omega$ is the angular position of the contact line, measured vertically from the centre of the sphere. The forces acting on the sphere, i.e. the forces due to surface tension $\left(F_{\mathrm{st}}\right)$, hydrostatic pressure $\left(F_{\mathrm{hp}}\right)$ and the object's weight $\left(F_{\mathrm{w}}\right)$, are also indicated. (b) For a pair of floating spheres, the three-phase contact line is no longer flat so that $\omega$ is a function of $\alpha$.

method of solution based on the asymptotic analysis of the meniscus around a cylinder. ${ }^{3}$ This model is able to reproduce, and helps to explain, many of the features of the numerical results for the transition from floating to sinking that we observe. Finally, we compute the force of interaction between identical floating spheres and compare these results to previous asymptotic results.

\section{An isolated sphere}

We consider an isolated sphere of radius $R$ and density $\rho_{s}$ floating at the interface between a liquid, of density $\rho_{l}$, and a fluid, of density $\rho_{f}$. The three phases, solid, liquid and fluid, meet at the contact line with a constant contact angle $\theta$; the angular position of the contact line is denoted by $\omega$. The geometry and our notation are summarized in figure 1(a). The problem is axisymmetric and so $\omega$ is constant - the meniscus is a planar circle. This axisymmetry simplifies the problem allowing approximate analytic solutions to be developed. ${ }^{10-12}$ The first step in doing this is to solve for the meniscus profile around a cylindrical object (since the 
axisymmetric contact line around a floating sphere is indistinguishable from that around an equivalent cylinder).

The profile of the meniscus is given by equating the hydrostatic pressure at each side of the interface with the pressure jump due to the interfacial tension, $\gamma$; we denote the interface position by $z=\zeta(r)$ where $r=\left(x^{2}+y^{2}\right)^{1 / 2}$ is the radial coordinate and find that $\zeta(r)$ satisfies the Laplace-Young equation

$$
\left(\rho_{l}-\rho_{f}\right) g \zeta=\gamma \frac{1}{r} \frac{\mathrm{d}}{\mathrm{d} r}\left[\frac{r \zeta_{r}}{\left(1+\zeta_{r}^{2}\right)^{1 / 2}}\right]
$$

where $\zeta_{r}=\mathrm{d} \zeta / \mathrm{d} r$. The Laplace-Young equation (1) is to be solved subject to the boundary conditions that $\zeta(r) \rightarrow 0$ as $r \rightarrow \infty$ and the requirement that the interfacial inclination is some angle $\psi$ at the contact line, i.e. that

$$
\left.\frac{\mathrm{d} \zeta}{\mathrm{d} r}\right|_{r=R \sin \omega}=\tan \psi
$$

Although we have specified two boundary conditions for the second-order differential equation (1), the problem is not yet closed since the angles $\psi$ and $\omega$ are not yet determined. Elementary geometry, together with the assumption of a constant contact angle $\theta$ gives us that

$$
\psi=\theta-\omega,
$$

so that only $\omega$ remains undetermined.

The final condition, required to determine $\omega$, arises from the requirement that the vertical forces on the sphere balance. (Heuristically, this can be seen be imagining the case of a very dense sphere, for which we might expect $\omega$ to be small, and the case of a very light sphere, for which we might expect $\omega$ to be close to $\pi$. We therefore see that the position of the contact line must depend on the weight of the object.)

Calculating the surface integral of the vertical component of the hydrostatic pressure on 
the sphere, we find that the net vertical hydrostatic pressure acting upwards on the sphere $F_{\mathrm{hp}}^{(z)}$ is:

$$
F_{\mathrm{hp}}^{(z)}=\frac{4}{3} \pi R^{3} \rho_{f} g+\pi R^{3}\left(\rho_{l}-\rho_{f}\right) g\left(\frac{2}{3}+\cos \omega-\frac{1}{3} \cos ^{3} \omega-\frac{\zeta_{m}}{R} \sin ^{2} \omega\right)
$$

where $\zeta_{m}=\zeta(R \sin \omega)$ is the vertical position of the contact line. We note that this result is equivalent to that given previously ${ }^{12}$ using the "generalized Archimedes principle", 30,31 which states that the total vertical force on a floating object is equal to the total weight of liquid $l$ displaced by the object including that displaced in the meniscus around it. The force due to surface tension is equal to the weight of liquid displaced in the meniscus while the remainder is equal to that displaced by the body itself, which is equal to that given in (4). For our purposes, it is simpler to calculate the surface tension force as that directed tangential to the interface and of strength $\gamma ;{ }^{12,31}$ we find that

$$
F_{\mathrm{st}}^{(z)}=2 \pi \gamma R \sin \omega \sin \psi
$$

The final force to be considered in the force balance is the weight of the sphere itself, $F_{\mathrm{w}}$, which is given by

$$
F_{\mathrm{w}}=\frac{4}{3} \pi R^{3} \rho_{s} g
$$

In equilibrium, these forces must balance. To write this force balance as simply as possible, we introduce the dimensionless sphere density ratio $D=\left(\rho_{s}-\rho_{f}\right) /\left(\rho_{l}-\rho_{f}\right)$, the sphere Bond number Bo $=\left(\rho_{l}-\rho_{f}\right) g R^{2} / \gamma$ (which gives a dimensionless sphere radius $\mathrm{Bo}^{1 / 2}$ ) and the capillary length $\ell_{c}=\left[\gamma /\left(\rho_{l}-\rho_{f}\right) g\right]^{1 / 2}$, which measures the typical horizontal length scale over which the meniscus becomes flat. We find that the vertical force balance may then be 
written

$$
\frac{4}{3} D=\frac{2}{3}+\cos \omega-\frac{1}{3} \cos ^{3} \omega-\frac{\zeta_{m}}{\mathrm{Bo}^{1 / 2} \ell_{c}} \sin ^{2} \omega+2 \mathrm{Bo}^{-1} \sin \omega \sin (\theta-\omega)
$$

Here the first term on the RHS arises from $F_{\mathrm{hp}}$, while the second comes from $F_{\text {st }}$ and dominates for small spheres, Bo $\ll 1$.

We note that the meniscus height, $\zeta_{m}$, may be determined, for given values of $\omega$, Bo $=$ $R^{2} / \ell_{c}^{2}$ and $\theta$, by solving the differential equation (1) subject to the boundary condition (2). We may then view the vertical force balance (7) as an equation for the sphere density $D$ that is required to reach a given contact line position $\omega$ for given sphere properties Bo and $\theta$. Viewed in this way, the function $D(\omega)$ has a maximum that corresponds to the maximum

density that such a sphere can have and float in equilibrium at the interface. ${ }^{10,12,29}$ While this maximum value may readily be calculated numerically for arbitrary $\theta$ and Bo, it is only possible to make analytical progress in the limit Bo $\ll 1$ for which the final term on the RHS of (7) dominates; the maximum density occurs when $\omega \approx \theta / 2$ and we find that

$$
D_{\max }^{(1)} \approx \frac{3}{2 \mathrm{Bo}} \sin ^{2} \frac{\theta}{2}
$$

where $D_{\max }^{(1)}$ is the maximum density of an isolated sphere that can float. This result will be a useful benchmark against which to test our numerical code for the problem of two floating spheres. We now turn to the setting up of the two-body problem.

\section{Two Spheres: Theoretical setting}

\section{Geometrical considerations}

Figure 1(b) shows a pair of spheres floating at a liquid-fluid interface with a centre-centre separation, $d$. The key complication in this case over that of the isolated sphere just considered is that axisymmetry is broken and so the contact lines are no longer planar and 
circular. In principle, it might be expected that this complicated geometry could lead to a resultant torque on the sphere (giving an extra balance that needs to be satisfied in addition to vertical force balance). However, it has been shown previously ${ }^{32}$ that for spheres with a constant contact angle floating at an interface with constant surface tension, the resultant torque due to interfacial forces is always zero. Nevertheless, the non-trivial shape of the contact line makes satisfying the boundary conditions when calculating the interface shape and calculating the forces exerted by the fluids on the spheres more complex than is the case for the single sphere case.

In this scenario, it is useful to refer to spherical coordinates $(r, \alpha, \phi)$ centred on each of the spheres. In this notation, the position of the contact line is given by $\phi=\omega(\alpha)$, and the normal and tangent vectors, $\mathbf{n}$ and $\mathbf{t}$ can be computed in the usual way (see Supporting Information A for details). The unit bi-normal $\hat{\mathbf{b}}$ is given in terms of the unit normal and tangent (denoted $\hat{\mathbf{n}}$ and $\hat{\mathbf{t}}$, respectively) by $\hat{\mathbf{b}}=\hat{\mathbf{n}} \times \hat{\mathbf{t}}$.

The contact angle $\theta$ is defined in the plane containing $\boldsymbol{b}$ and $\boldsymbol{n}$, so that

$$
\hat{\boldsymbol{b}} \cdot \hat{\gamma}=\cos \theta,
$$

where $\hat{\boldsymbol{\gamma}}$ is the unit tangent to the liquid interface in the $(\hat{\boldsymbol{b}}, \hat{\boldsymbol{n}})$ plane.

\section{Vertical balance for floating}

For the spheres to be floating at the liquid-fluid interface, the vertical forces on them must balance. The net vertical force on an individual sphere may be written

$$
N=F_{\mathrm{st}}^{(z)}+F_{\mathrm{hp}}^{(z)}-F_{\mathrm{w}},
$$

so that in equilibrium $N=0$.

Integrating to find the resultant vertical component of the hydrostatic pressure, we find 
that

$$
F_{\mathrm{hp}}^{(z)}=\frac{4 \pi}{3} \rho_{f} g R^{3}+\Delta \rho g R^{3} \int_{0}^{2 \pi}\left\{\frac{1}{3}\left[1+\cos ^{3} \omega(\alpha)\right]-\frac{z_{c}}{2 R} \sin ^{2} \omega(\alpha)\right\} \mathrm{d} \alpha,
$$

where $z_{c}$ is the vertical position of the centre of the sphere and $\Delta \rho=\rho_{l}-\rho_{f}$. (Note that this result reduces to $(4)$ when $\omega(\alpha)=\omega$, a constant, upon using the geometrical result $\left.\zeta_{m}=z_{c}+R \cos \omega.\right)$

The calculation of the vertical force from surface tension uses some involved geometry; details are given in Supporting Information B. The resultant vertical force on a sphere may be written

$$
\begin{aligned}
N= & \gamma R \int_{0}^{2 \pi}\left[\lambda \cos \omega(\alpha) \sin \theta-\sin ^{2} \omega(\alpha) \cos \theta\right] \mathrm{d} \alpha \\
& +R^{3} \Delta \rho g \int_{0}^{2 \pi}\left\{\frac{1}{3}\left[1+\cos ^{3} \omega(\alpha)\right]-\frac{z_{c}}{2 R} \sin ^{2} \omega(\alpha)\right\} \mathrm{d} \alpha \\
& -\frac{4}{3} \pi R^{3}\left(\rho_{s}-\rho_{f}\right) g,
\end{aligned}
$$

where

$$
\lambda=\left[\sin ^{2} \omega+\left(\frac{\mathrm{d} \omega}{\mathrm{d} \alpha}\right)^{2}\right]^{1 / 2}
$$

is a normalization factor. We shall see that determining the value of $N$ for a given contact line shape $\omega(\alpha)$ is a convenient way of determining whether a sphere is floating in equilibrium or not.

\section{Interface profile}

As for the case of an isolated sphere, the resultant vertical force on a sphere, given by (12) can only be determined once the shape of the liquid-fluid interface, $z=\zeta(x, y)$, has been determined. Here, because the system is no longer axisymmetric, we work in Cartesian 
coordinates centred on the sphere. The interface profile, $\zeta(x, y)$, must satisfy the LaplaceYoung equation

$$
\zeta=\ell_{c}^{2} \kappa,
$$

where $\kappa$ is the interfacial curvature given by ${ }^{33}$

$$
\kappa=\frac{\zeta_{x x}\left(1+\zeta_{x}^{2}\right)+\zeta_{y y}\left(1+\zeta_{y}^{2}\right)-2 \zeta_{x} \zeta_{y} \zeta_{x y}}{\left(1+\zeta_{x}^{2}+\zeta_{y}^{2}\right)^{\frac{3}{2}}}
$$

The boundary condition for the meniscus in contact with the surface of a sphere is a fixed contact angle, which is defined in (9). This is equivalent to

$$
\hat{\boldsymbol{n}} \cdot \hat{\boldsymbol{s}}=\cos \theta,
$$

where $\hat{\boldsymbol{s}}$ is the outward unit normal vector to the liquid-fluid interface from a point on the contact line along the plane containing $\hat{\boldsymbol{b}}$ and $\hat{\boldsymbol{n}}$, given by

$$
\hat{\boldsymbol{s}}=\frac{\zeta_{x} \boldsymbol{e}_{\boldsymbol{x}}+\zeta_{y} \boldsymbol{e}_{\boldsymbol{y}}-\boldsymbol{e}_{z}}{\sqrt{\zeta_{x}^{2}+\zeta_{y}^{2}+1}}
$$

Substitution of the expressions for $\hat{\boldsymbol{s}}$, Eq. (17), and for $\hat{\boldsymbol{n}}$, Eq. (??) in supporting information, into Eq. (16) yields the boundary condition for the derivatives of $\zeta$

$$
\zeta_{x} \cos \alpha+\zeta_{y} \sin \alpha=\frac{\cos \omega-\cos \theta \sqrt{\zeta_{x}^{2}+\zeta_{y}^{2}+1}}{\sin \omega} .
$$

The second boundary condition on $\zeta$ is that

$$
\zeta(x, y) \rightarrow 0
$$

as $x, y \rightarrow \infty$.

Implementing the boundary condition Eq. (18) is not straightforward, because $\omega$ and 
the location of the boundary are also unknown. In the absence of an analytical solution, a numerical scheme is necessary to solve the two sphere problem. We shall shortly present a numerical scheme designed to solve the Laplace-Young equation (14) subject to the boundary conditions (18) and (19) whilst also taking into account the vertical force balance condition (12). However, we first present a new analytical approach that allows us to approximately take into account the effect of the distance, $d$, between two particles.

\section{Approximate solution}

In this section, we develop approximations for the vertical forces of hydrostatic pressure and surface tension as functions of the centre-centre separation $d$ and determine how the changes of these forces affect $D_{\max }^{(2)}$ - the maximum density that a pair of spheres can have and remain floating at a liquid-fluid interface.

\section{Increase of the hydrostatic pressure force}

When a sphere is floating at a liquid-fluid interface and a second identical sphere is placed a distance $d$ away from the first sphere, the meniscus created by the second sphere moves the first sphere vertically downwards (and vice versa). This downwards movement increases the vertical force of hydrostatic pressure exerted on each sphere. In this section, we calculate this additional force using an approximation for the vertical movement of the spheres.

The meniscus around a sphere with a flat contact line is identical to the meniscus around a vertical cylinder of radius $R \sin \omega$ and meniscus slope angle $\psi=\theta-\omega$. Far from such a

cylinder, the meniscus deflection is given by $\zeta(r)=-R \sin \omega \sin (\theta-\omega) K_{0}\left(r / \ell_{c}\right),{ }^{3,22}$ where $K_{0}(x)$ is the zeroth order modified Bessel function of the second kind. ${ }^{34}$ Although this expression is only strictly relevant for a single, isolated sphere, an attempt to account for the presence of a second sphere, identical to the first but with centre-to-centre separation $d$, 
can be made by assuming that this result remains valid, i.e. that

$$
\zeta(r)=-R \sin \omega_{s} \sin \left(\theta-\omega_{s}\right) K_{0}\left(r / \ell_{c}\right)
$$

where $\omega_{s}$ denotes the value of $\omega$ for an isolated sphere. Linear superposition of the individual menisci shows that the vertical movement of a sphere due to the presence of second identical sphere at a distance $d$ away is $-R \sin \omega \sin \left(\theta-\omega_{s}\right) K_{0}\left(d / \ell_{c}\right)$. This vertical downwards movement increases the hydrostatic pressure (buoyancy) force acting on the sphere, enabling it to support an additional load. This may mean that the relative density of the spheres can increase, while remaining afloat.

Although the presence of the second sphere changes the shape of the contact line on the first sphere, we neglect this effect when calculating the buoyancy force which is more significantly affected by the vertical position of the sphere than the tilting of the contact line. We consider the changes to contact line shape only when calculating the surface tension force, which will be described later. The downwards movement of the sphere makes the upper fluid displace a cylindrical volume of the lower liquid with radius $R \sin \omega$ and height $R \sin \omega_{s} \sin \left(\theta-\omega_{s}\right) K_{0}\left(d / \ell_{c}\right)$. This results in an additional hydrostatic pressure force of

$$
F_{\mathrm{hp}, \text { additional }}^{(z)}=\pi\left(R \sin \omega_{s}\right)^{2} \times R \sin \omega_{s} \sin \left(\theta-\omega_{s}\right) K_{0}\left(d / \ell_{c}\right) \Delta \rho g .
$$

Considering this change in the force, the maximum relative density of a pair of floating spheres can be obtained as a function of the inter-particle distance:

$$
\begin{aligned}
D_{\max }^{\mathrm{hp}}(d) & =D_{\max }^{(1)}+\frac{F_{\mathrm{hp}, \text { additional }}^{(z)}}{4 / 3 \pi R^{3} \rho_{w} g} \\
& =D_{\max }^{(1)}+\frac{3}{4} \frac{\Delta \rho}{\rho_{w}} \sin ^{3} \omega_{s} \sin \left(\theta-\omega_{s}\right) K_{0}\left(d / \ell_{c}\right) .
\end{aligned}
$$

To carry out this calculation in practice, the maximum density of an isolated floating sphere, $D_{\max }^{(1)}$, and $\omega_{s}$ are calculated numerically using the theory presented previously. 


\section{Reduction of the surface tension force}

When a floating sphere is part of a pair, its contact line is no longer planar, as already discussed. We calculate the contact line height $\zeta_{m}$ of the first sphere by linearly superposing the meniscus created by the second sphere. In doing this, the geometry of the sphere is neglected and the contact line is assumed to move only vertically:

$$
\zeta_{m, p}(\alpha)=\zeta_{m, s}-R \sin \omega_{s} \sin \left(\theta-\omega_{s}\right) K_{0}\left[r(\alpha) / \ell_{c}\right]
$$

where $\zeta_{m, s}$ is the contact line height of a single (isolated) sphere, $\zeta_{m, p}$ is the contact line height when another identical sphere is present, and $r(\alpha)$ is the horizontal distance from the centre of one sphere to a point on the contact line of the other sphere and is obtained using Pythagoras' theorem

$$
r(\alpha)=\left[d^{2}+R^{2} \sin ^{2} \omega_{s}-2 d R \sin \omega_{s} \cos \alpha\right]^{1 / 2}
$$

Since the angular position of the contact line, $\omega$, is related to the contact line height $\zeta$ and the position of the sphere's centre, $z_{c}$, we have that

$$
\omega=\arccos \left(\frac{\zeta_{m}-z_{c}}{R}\right)
$$

Hence, for a pair of identical spheres, the angular position of the contact line $\omega_{p}(\alpha)$ is given, according to the above approximation, by

$$
\begin{aligned}
\omega_{p}(\alpha) & =\arccos \left(\frac{\zeta_{m, s}-R \sin \omega_{s} \sin \left(\theta-\omega_{s}\right) K_{0}\left[r(\alpha) / \ell_{c}\right]-z_{c, s}+R \sin \omega_{s} \sin \left(\theta-\omega_{s}\right) K_{0}\left(d / \ell_{c}\right)}{R}\right) \\
& =\arccos \left\{\cos \omega_{s}-\sin \omega_{s} \sin \left(\theta-\omega_{s}\right)\left\{K_{0}\left[r(\alpha) / \ell_{c}\right]-K_{0}\left(d / \ell_{c}\right)\right\}\right\}
\end{aligned}
$$

where the subscript $p$ denotes the presence of a pair of spheres, $r(\alpha)$ is given by $(24)$, and $z_{c, s}$ is the vertical location of the centre of an isolated sphere. 
We are able to compute (by substituting (26) into results for the surface tension force given in Supporting Information B) the additional surface tension force acting on the first sphere as a result of the presence of the second sphere as an integral

$$
\begin{aligned}
F_{\text {st, additional }}^{(z)}= & \gamma R \int_{0}^{2 \pi}\left[\lambda \cos \omega_{p}(\alpha) \sin \theta-\sin ^{2} \omega_{p}(\alpha) \cos \theta\right] \mathrm{d} \alpha \\
& -2 \pi R \gamma \sin \omega_{s} \sin \left(\theta-\omega_{s}\right),
\end{aligned}
$$

where $\lambda$ is obtained by substituting $\omega_{p}$ into (13). Similarly to the additional force due to buoyancy, (22), the change of surface tension force changes the maximum density of a pair of spheres that can float:

$$
D_{\max , \text { st }}(d)=D_{\max }^{(1)}+\frac{F_{\text {st, additional }}^{(z)}}{4 / 3 \pi R^{3} \rho_{w} g}
$$

We shall see subsequently that $F_{\text {st, additional }}^{(z)}$ is in fact negative at close range: the change in the contact line shape results in a reduction of the density of sphere that is able to float.

\section{Combination of the two effects}

Combination of (22) and (28) gives the maximum density of a pair of spheres that can float:

$$
\begin{aligned}
D_{\max }^{(2)}(d)= & D_{\max }^{(1)}+\frac{F_{\mathrm{hp}, \text { additional }}^{(z)}+F_{\mathrm{st}, \text { additional }}^{(z)}}{4 / 3 \pi R^{3} \rho_{w} g} \\
= & D_{\max }^{(1)}+\frac{3}{4} \frac{\Delta \rho}{\rho_{w}} \sin ^{3} \omega \sin \left(\theta-\omega_{s}\right) K_{0}\left(d / \ell_{c}\right) \\
& +\frac{F_{\mathrm{st}, \text { additional }}^{(z)}}{4 / 3 \pi R^{3} \rho_{w} g} .
\end{aligned}
$$

We shall see (figure 4) that $D_{\max }^{(2)}(d)$ gives a good approximation of the numerical results for the full problem. However, we delay further discussion of the results until after we have presented this numerical scheme. 


\section{Numerical solution of the full problem}

\section{The numerical scheme}

The Laplace-Young equation (14) with the boundary conditions (18) and (19) as well as the requirement that the vertical forces on each sphere balance constitute a free boundary problem since the contact line position $\omega(\alpha)$ and $z_{c}$ are unknown. We solve this problem numerically using an iterative method. Details of the iteration scheme are given in Supporting Information $\mathrm{C}$. In short, we initially guess values for the contact line position $\omega(\alpha)$ and the sphere centre height, $z_{c}$. (In practice, we select $\omega(\alpha)=\theta / 2$, which approximates the value for the most dense isolated sphere in equilibrium, and $z_{c}=-R \cos \omega$ as the starting guess.) With these variables given, we then solve the Laplace-Young equation using the $h p$-meshless cloud method, ${ }^{22,35}$ which is a meshfree finite difference method. (The meshless cloud method uses an array of nodes distributed in a domain of interest between the contact line and the edge of the domain, at " $\infty$ "; we find that an outer domain at $r=7 \ell_{c}$ was sufficient for our purposes. Details of the distribution of nodes are given in. ${ }^{36}$ )

The Laplace-Young equation Eq. (14) and the boundary condition Eq. (18) are both nonlinear equations. To handle this nonlinearity, the meniscus height at the $(N-1)^{\text {st }}$ iteration is used to give estimates of all nonlinear terms so that a linear equation is obtained for the interface profile at the $N^{\text {th }}$ iteration.

Once this iteration scheme has converged, we have the meniscus shape around a sphere, albeit with an incorrect contact line shape and incorrect sphere centre position (i.e. the sphere is not in general in equilibrium). To rectify this, we take the meniscus height around the contact line, $\zeta_{m}(\alpha)$, and modify the sphere height, $z_{c}$, taking care to modify the angular position of the contact line, $\omega(\alpha)$, in such a way that $\zeta_{m}(\alpha)$ is fixed. We therefore use a new contact line position

$$
\omega(\alpha)=\arccos \left[\frac{\zeta_{m}(\alpha)-z_{c}}{R}\right]
$$

with $z_{c}$ in the above equation changing and $\zeta_{m}(\alpha)$ fixed. Using $z_{c}$ and the resulting $\omega(\alpha)$, 
the net force $N$ acting on the sphere may be calculated using Eq. (12); $z_{c}$ is taken as the value that minimizes $|N|$. In some cases, this minimum value of $N$ can be zero, while in other instances there may be two values for $z_{c}$ for which $N=0$. It is also possible for $N=0$ to have no solutions at all. Since $N$ is the net force acting upwards and $z_{c}$ is a distance measured downwards, only a solution with

$$
\frac{\partial N}{\partial z_{c}}<0
$$

corresponds to a stable equilibrium of the sphere, consistent with what was presented for an isolated sphere. ${ }^{10}$ However, when there are no solutions of $N=0$, we only find a solution with

$$
\frac{\partial N}{\partial z_{c}}=0
$$

i.e. a minimum of the resultant force $N$. In order to force the algorithm to converge on a stable equilibrium wherever possible, we set the general criterion for selecting a value for $z_{c}$ to be minimizing $|N|$ with

$$
\frac{\partial N}{\partial z_{c}} \leq 0
$$

$N_{\text {res }}$ is the residual value of $N$ after this minimization. Now using the new $z_{c}$ and $\omega(\alpha)$, we repeat the iterations until the difference between each of $z_{c}$ and $\omega(\alpha)$ are acceptably small between two consecutive steps. An algorithm summarizing this process is given in Supporting Information C.

In our numerical solutions for pairs of spheres, only identical spheres were considered. Both spheres were included in the solution of the Laplace-Young equation but only one sphere was considered in determining its equilibrium position and contact line and the configuration of the second sphere was determined using the symmetry of the system. 


\section{Validation of the numerical scheme}

A simple scenario in which the above numerical scheme may be validated is the flotation of a single, isolated sphere. Figure 2 shows the values of $N_{\text {res }}$ obtained using the numerical solution outlined above for a sphere with a fixed radius and contact angle, but various densities $D$. In such a simple scenario, the theory for an isolated sphere predicts that floating should only be possible for $D<D_{\max }^{(1)}$ (for the particular parameters used in the simulations of figure $2, D_{\max }^{(1)} \approx 11.28$ ). In fig. 2 we see that for $D<D_{\max }^{(1)}$ the numerically determined value of $N_{\text {res }}$ is either exactly 0 or 0 to within machine precision. However, for $D>D_{\max }^{(1)}$ our numerical code is only able to find a minimal value of $|N|$ so that $N_{\text {res }}$ takes a finite, non-zero, value. We interpret this failure to find an equilibrium floating scenario as the onset of sinking. We also note that in the numerical approach we adopt here, sinking is characterised by a sharp increase in the value of $N_{\text {res }}$ away from machine precision. We now go on to investigate the flotation behavior of two identical spheres.

\section{Results}

Figure 3(a) shows the variation of $N_{\text {res }}$ with the centre-centre separation for two identical spheres with density slightly larger than $D_{\max }^{(1)}$ - the maximum density at which an isolated sphere can float. We see that for large separations $d \gg \ell_{c}, N_{\text {res }}$ becomes large: the two spheres sink. This is as might be expected since, when separated by a large distance, the spheres are effectively isolated and, since $D>D_{\max }^{(1)}$, they cannot float in equilibrium. However, for $d / \ell_{c} \lesssim 1.5$ we see that $N_{\text {res }}$ is zero to within machine precision, i.e. the spheres are able to float at intermediate range. We see this surprising behavior resulting from both the full numerical model and the approximate model.

As $d / \ell_{c}$ decreases further the full numerical code shows that close to contact the value of $N_{\text {res }}$ increases again, corresponding to the spheres sinking. This behavior is also predicted by our approximate model.

We note that the transition from floating to sinking at very small centre-centre separation 
Figure 2: Minimum residual net force, $N_{\text {res }}$, calculated as a function of sphere density, $D$, for a sphere with $R / \ell_{c}=0.2209$ and $\theta=70^{\circ}$. For these parameters, $D_{\max }^{(1)}=11.2826$. Large points represent the results of the numerical algorithm described in this section and Supporting Information $\mathrm{C}$ with results where $N_{\text {res }}=0$ represented by $\boldsymbol{\nabla}$ and results with minimal values of $N_{\text {res }}$ given by $\bullet$. We see that the onset of sinking with $D>D_{\max }^{(1)}$ is signified by a rapid increase in the residual $N_{\text {res }}$.

is consistent with previous results for the flotation of cylinders floating horizontally at an interface. ${ }^{23}$ In that two-dimensional problem, short range sinking was attributed to the significant tilting of the contact line as the objects approach one another, reducing the vertical supporting force that can be supplied by surface tension. This is again observed here (see figure 3). However, the observation that two dense spheres may float nearby one another even if their density is larger than $D_{\max }^{(1)}$ is novel and deserves further investigation. The interfacial cross sections in figure 3(b) and sketches in figure 3(c) illustrate the reason for this behavior. As the spheres move closer to one another, they move vertically downwards due to the meniscus created by the other sphere. This increases the buoyancy force acting on each sphere, allowing them to float at higher densities than would otherwise be possible. The vertical components of the hydrostatic and surface tension forces acting on the spheres in the floating regime are plotted in figure 3(d). This clearly shows the increase of the hydrostatic 

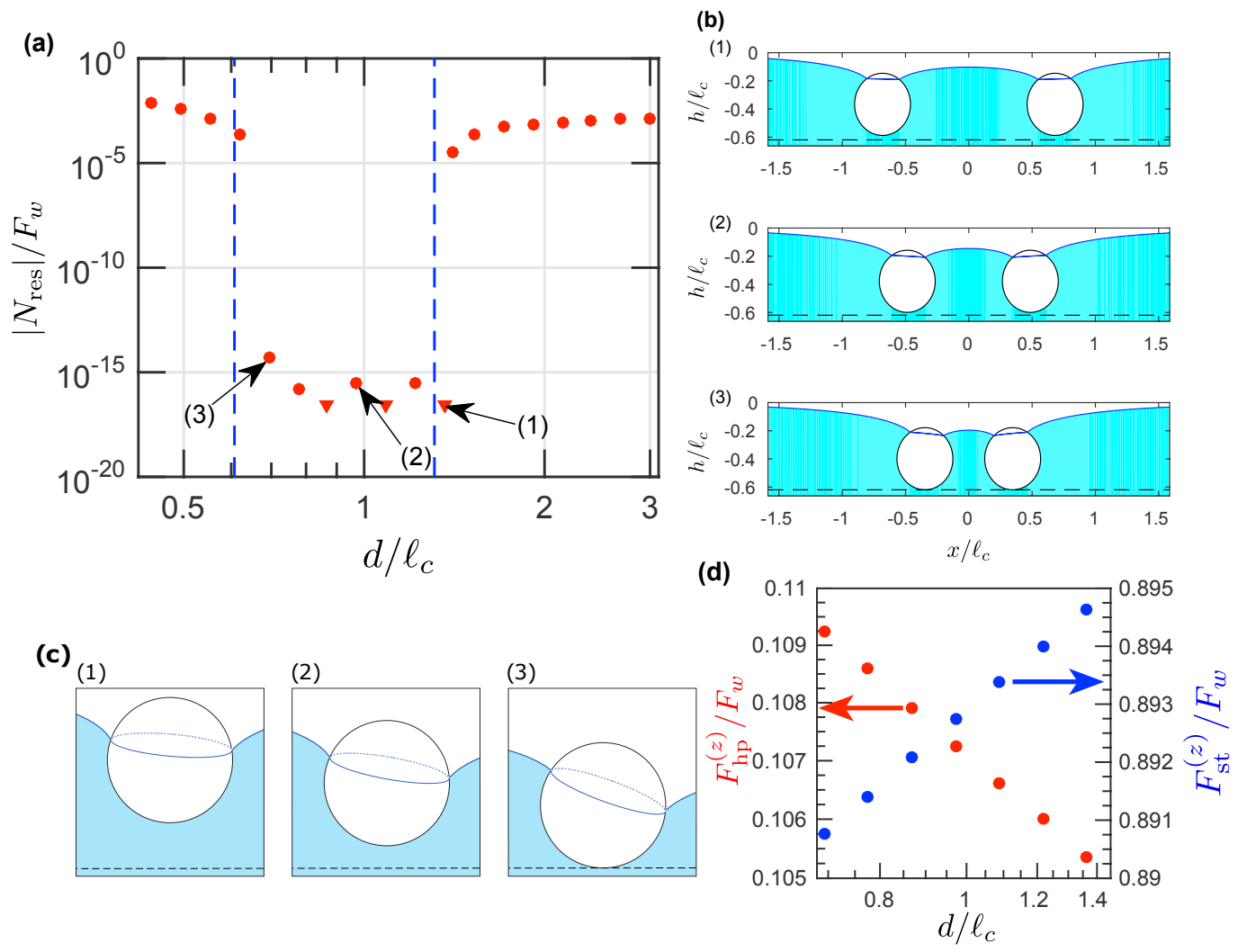

Figure 3: (a) Numerically determined residual vertical forces, $N_{\text {res }}$, as a function of distance $d$ between two identical spheres with $R / \ell_{c}=0.2209, D=11.3015$ and $\theta=70^{\circ}$. For these parameters, the maximum density of an isolated sphere is $D_{\max }^{(1)}=11.2826$ and so these two spheres sink at large distances (signified by a non-zero value of $N_{\text {res }}$ ). However, there is an interval of intermediate distances for which the spheres are able to float as a pair. Results are shown for the numerical solution of the full problem and the approximate solution. The results from the full numerical solution are denoted by symbols depending on whether $N_{\text {res }}=0(\mathbf{v})$ or simply that $N_{\text {res }}$ is minimized $(\bullet)$. The blue dashed lines (--) show the boundaries of the intermediate floating regime given by the approximate solution (29). (b) Cross sections along the principal axis of the liquid-air interface for the pair of spheres shown in panel (a), in the regime where they float. Panels (b.1), (b.2) and (b.3) are for the correspondingly marked locations in panel (a). The black dashed lines (- -) denote the lowest position reached by the spheres at the closer end of the intermediate floating regime. The reasons for the floating and sinking regimes are illustrated by the schematic diagrams in panel (c). As the spheres approach each other (c.1 to c.3), their centres move downwards. This increases the vertical force from hydrostatic pressure $\left(F_{\mathrm{hp}}^{(z)}\right)$, as shown in the red symbols $(\bullet)$ in panel (d), enabling them to support a higher load. As they move even closer, the their contact lines become more tilted; this reduces the surface tension component of the vertical force $\left(F_{\mathrm{st}}^{(z)}\right)$ as also seen for floating cylinders. ${ }^{23}$ This is shown by the blue symbols $(\bullet)$ in panel (d). 

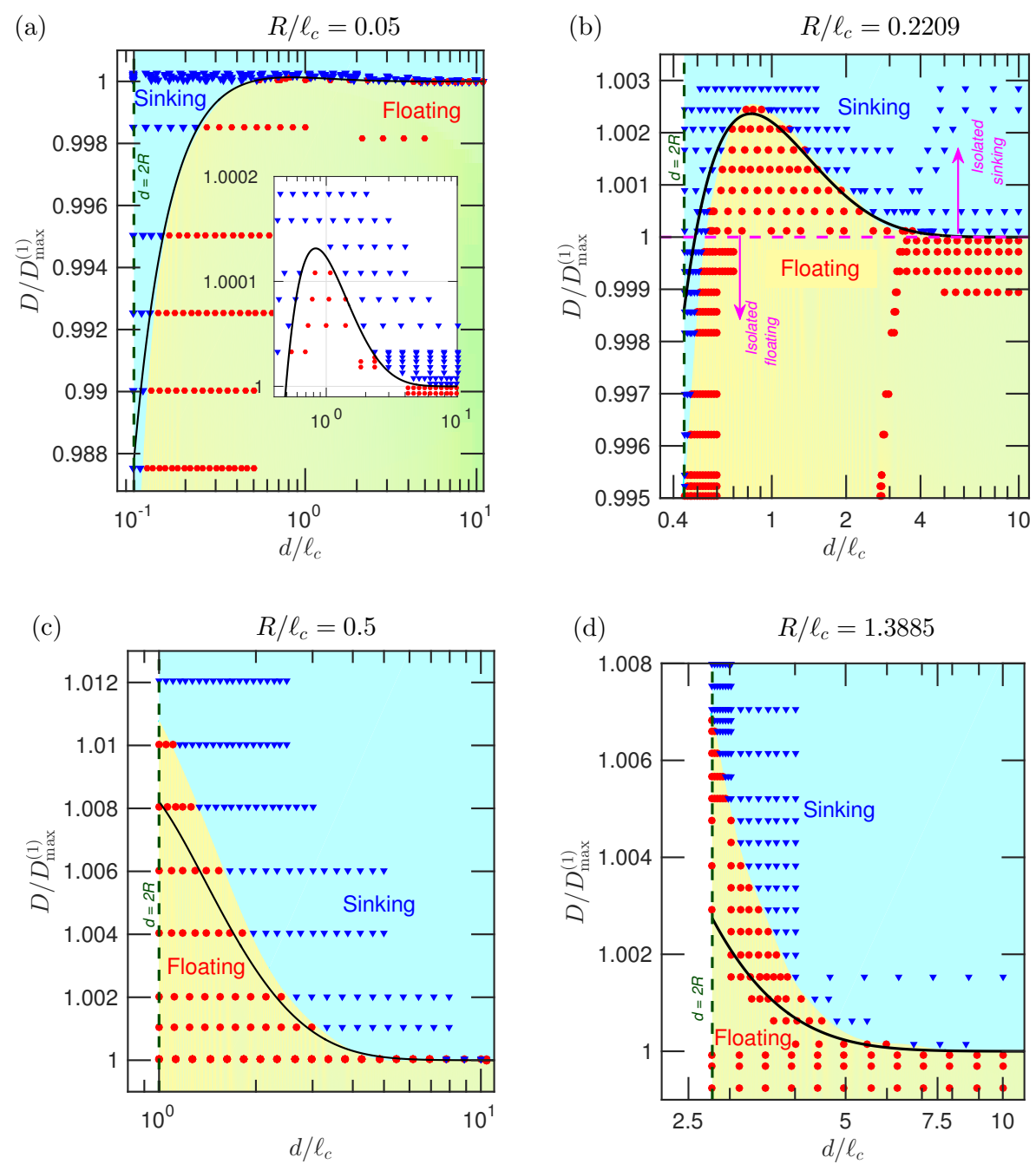

Figure 4: Regime diagrams showing the sinking and floating behavior for pairs of identical spheres showing the regions of separation-density space for which the pair of spheres float and sink. The symbols show points in this space for which the full numerical solution suggests the spheres will float $(\bullet)$ and $\operatorname{sink}(\mathbf{v})$. The black solid curves $(-)$ shows $D_{\max }^{(2)}(d)$ calculated using the approximate solution (29) for which $D_{\max }^{(1)}$ and $\omega_{s}$ obtained from the numerical solution were used as input parameters. The dark green vertical dashed lines denote the location where the spheres touch. All the spheres have the same contact angle $\left(\theta=70^{\circ}\right)$ with only the radii variable. The radii (and associated maximum isolated densities) are as follows: (a) $R / \ell_{c}=0.05, D_{\max }^{(1)}=198.6703$, (b) $R / \ell_{c}=0.2209, D_{\max }^{(1)}=11.2826$, (c) $R / \ell_{c}=0.5$, $D_{\max }^{(1)}=3.0812$, and $(\mathrm{d}) R / \ell_{c}=1.3885, D_{\max }^{(1)}=1.3031$. All the regime diagrams show that a sphere that is too heavy to float in isolation may float as part of a pair. Although the relative significance of this "intermediate floating" reduces as $R / \ell_{c} \rightarrow 0$, the detailed inset in (b) shows that this effect is still present in small spheres as well. (a) and (b) also show that a sphere that can float in isolation can sink if another sphere is present at very close range $\left(d / \ell_{c} \rightarrow 0\right)$. In contrast to the "intermediate floating" effect, this "close range sinking" effect is not present in spheres that are large (compared to the capillary length, $\ell_{c}$ ) because such spheres touch each other at the equators, keeping their contact lines relatively far apart. The approximate solution (29) is plotted as the solid black curve in each case, and can approximate both "intermediate floating" and "close range sinking" regimes very well, particularly for small spheres. 


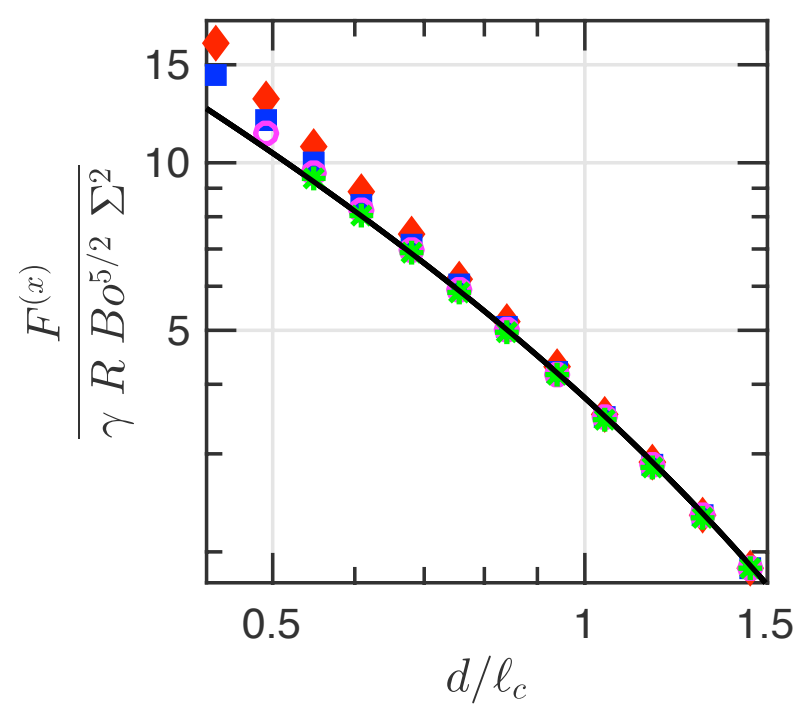

Figure 5: The horizontal force of capillary attraction between a pair of identical spheres with $R / \ell_{c}=0.2209$ and $\theta=70^{\circ}$. For these parameters, $D_{\max }^{(1)}=11.2826$. The results obtained using the full numerical solution are shown by symbols for spheres with different relative densities: $D=0.99 D_{\max }^{(1)}(\bullet), D=0.75 D_{\max }^{(1)}(\mathbf{\square}), D=0.5 D_{\max }^{(1)}(\circ)$ and $D=0.25 D_{\max }^{(1)}$ $(*)$. The solid curve is (35) that results from the solution of the linearized Laplace-Young equation and linear superposition of the menisci. ${ }^{11}$ When the density of the sphere is small, the approximate solution agrees very well with the full numerical solution. However, as $D \rightarrow D_{\max }^{(1)}$, the this approximation loses its accuracy at close range.

pressure force and decrease of the surface tension force as the spheres approach each other.

To test the dependence of the magnitude of the floating and sinking regimes on the sphere size, and to summarize the new finding of floating at intermediate separations, we show in figure 4 regime diagrams for different values of $R / \ell_{c}$ showing when the sinking and floating occurs. The figure shows that the "intermediate floating" effect diminishes as the sphere radius gets small $\left(R \ll \ell_{c}\right)$. Since it is the effect of buoyancy that is providing the additional supporting force that leads to this effect, such a mechanism would not allow a significant increase in supporting force for very small spheres where the supporting force is predominantly provided by surface tension. Instead, dense, small spheres $\left(R \ll \ell_{c}\right) \operatorname{sink}$ as they come close to one another since the meniscus tilting that occurs is more detrimental for the spheres' ability to float than the additional force from buoyancy is beneficial.

The regime boundaries in figure 4 are very well described by our approximate solution (29), especially when $R \ll \ell_{c}$ and $d \gg R$. At very close range, this result deviates from the 
numerically obtained result because the errors produced by the linearization of the LaplaceYoung equation and linear superposition, and also because the meniscus profile given by (20) is a long-range asymptotic solution. However, even at short range, the simplified analytic solution qualitatively captures the correct behavior.

Figure 4 shows that the increase in maximum floating density for a pair of spheres (compared with an isolated sphere) is very small, on the order of a fraction of $1 \%$. We also see that the size of this effect increases with increasing sphere radius (the relevant dimensionless parameter being $\left.R / \ell_{c}\right)$. However, a pair of large spheres can touch each other while still keeping their contact lines well separated; we believe that this is the reason that the upper limits of the floating regimes in figure 4(c) and (d) do not show local maxima. If $\theta \approx \pi$, the contact lines will be closer to the equator according to (8), and so will be closer to one another as the spheres touch. We therefore expect the enhanced flotation effect to be more significant for large superhydrophobic spheres (i.e. $R / \ell_{c} \gtrsim 1$ ). Nevertheless, we suggest, based on fig. 4(c) and (d), that there is an optimal enhancement of the maximal flotation density achieved at intermediate sphere radius. Moreover, it may be possible for the magnitude of this effect to be more significant in the presence of more than two particles, i.e. in a cluster ${ }^{37}$ because the role of hydrostatic pressure is more significant in such a configuration.

At very close range, this result deviates from the numerically obtained result because the errors produced by the linearization of the Laplace-Young equation and linear superposition, and also because the meniscus profile given by (20) is a long-range asymptotic solution. However, even at short range, the simplified analytic solution qualitatively captures the correct behavior.

In addition to the above floating and sinking behavior, the deformation of a liquid interface by a pair of identical spheres also results in a horizontal force of capillary attraction - the Cheerios effect. ${ }^{13}$ The numerical solution of the interfacial shape enables us to determine this force as a function of the separation of the spheres by resolving the horizontal 
components of the surface tension and hydrostatic pressure forces. The surface tension force is given by Eq. (??) in supporting information and the horizontal hydrostatic pressure force can be calculated by integration in a way similar to (11) to obtain

$$
F_{\mathrm{hp}}^{(x)}=\frac{1}{6} \Delta \rho g R^{2} \int_{0}^{2 \pi}\left(3 z_{c} \cos \omega \sin \omega-2 R \sin ^{3} \omega-\omega\right) \cos \alpha \mathrm{d} \alpha
$$

The numerical results for the horizontal force of attraction $\left(F^{(x)}=F_{\mathrm{st}}^{(x)}+F_{\mathrm{hp}}^{(x)}\right)$ are plotted in figure 5. We compare these results with an approximate solution given in the literature, ${ }^{11}$ which is obtained by solving the linearized Laplace-Young equation and linear superposition of the menisci created by the spheres. According to this solution, the force of attraction is given by

$$
F^{(x)}=2 \pi \gamma R B o^{5 / 2} \Sigma^{2} K_{1}\left(d / \ell_{c}\right)
$$

where $K_{1}(x)$ is the first order modified Bessel function of the second kind ${ }^{34}$ and

$$
\Sigma=\frac{2}{3} D-\frac{1}{3}-\frac{1}{2} \cos \theta+\frac{1}{6} \cos ^{3} \theta
$$

Figure 5 shows that this solution is a good approximation to the numerical solution of the full interfacial profile for small particle densities and long-range interactions. However, heavy spheres at close-range involve large meniscus deformations; it is therefore unsurprising that in this regime the approximation loses its validity. We note that when this occurs, the nonlinear effects enhance the attractive force by around 30\%, similar to a related calculation in the two-dimensional case. ${ }^{38}$

\section{Conclusions}

A numerical solution was developed utilizing a new algorithm to accurately solve for the equilibrium of two identical floating spheres. In agreement with previous work on the floating of two-dimensional objects, ${ }^{23}$ we found that the ability to float is diminished when the objects 
come very close to contact because their contact lines are tilted, reducing the vertical force due to surface tension. Surprisingly, however, we also found an intermediate regime in which the flotation of dense particles may be slightly enhanced by the presence of other nearby particles. The mechanism of this enhanced flotation is the fact that particles of moderate radius lower themselves into the liquid in the presence of other particles, increasing the vertical force supplied by buoyancy, and so actually increase their ability to float. This behavior has not been discussed in the literature before. However, upon examining figure 2 of related work on floating cylinders, ${ }^{23}$ we notice a hint of similar behavior.

This increased load bearing capacity of interacting particles due to hydrostatic pressure is in competition with the well-established decrease in load-bearing capacity of the surface tension force as interacting particles come closer together. ${ }^{23,24,29}$ We have also calculated the horizontal force of attraction between pairs of spheres in this nonlinear regime. We find that the Nicolson approximation can underestimate the force of interaction in his regime by as much as $30 \%$, particularly for small, dense particles at small separation. Nevertheless, its accuracy in extreme circumstances where the approximation itself is not valid, is remarkable.

While practical interest is likely to focus on small Bond number, Bo $\ll 1$, for which it is the decrease in surface tension force that dominates, the observations of this paper highlight a new and potentially important aspect of these problems. We note that our numerical method can be generalized to the case of many floating particles all with the same vertical position. ${ }^{36}$ In this scenario, the effect described in this paper is more significant and leads to an enhancement of more than $30 \%$ in the density at which spheres may float (see figure 5.11 of Cooray $\left.{ }^{36}\right)$. In practical circumstances, the particles are most likely to float as a raft $^{24,25,37-39}$ and so all have different heights making practical computations significantly more challenging. Nevertheless, the preliminary results of Cooray ${ }^{36}$ on hexagonal lattices of particles with the same floating depth suggests that the effect described in this paper may be significant for floating rafts.

There are other situations where a rigid lattice of spheres located at a liquid-fluid interface 
is practically relevant, such as the forced entry or spontaneous (capillary) imbibition of a liquid into a fixed regular packing of spheres. ${ }^{40}$ These problems have similarities to the equilibrium of floating spheres at fixed liquid interfaces and so may be solved using the numerical method described here. In these problems, approximate solutions have already been derived under the assumption of uniform interfacial curvatures, ${ }^{41}$ but an accurate full solution is required to determine the surface tension and hydrostatic pressure forces, especially in situations where the contact lines have significant undulations. We also note that in the situations described here, the interface may become highly curved. While this curvature is captured by our numerical method, our assumption of a constant contact angle may no longer be valid: recent experiments suggest that even for surfaces with very small contact angle hysteresis (for an otherwise flat interface), the receding angle that is observed may vary significantly with increased curvature. ${ }^{42}$

Due to the horizontal force of capillary attraction, an experimental realisation of a full floating and sinking regime diagram requires an external means of keeping the spheres at a fixed given separation. However, we emphasize that it is the separation of the contact lines, and not the centre-to-centre distance of the spheres, that governs the neighbour-supported floating reported here. Therefore, although long-ranged repulsive forces can stabilize two particles at a finite separation (as seen with charged particles ${ }^{38}$ ), it is also possible for contact forces between touching spheres to provide this repulsion, while the contact lines remain separated: a pair of touching spheres can have their contact lines either almost touching or well separated, depending on their size and contact angle. Furthermore, such a pair can be in the close-range-sinking (see figure 4 (a) and (b)) or in the neighbour-supported floating (figure 4 (c) and (d)) regimes.

\section{Supporting Information Available}

- Geometrical considerations 
- Surface tension forces acting on the sphere

- Numerical Algorithm

This material is available free of charge via the Internet at http://pubs.acs.org/.

\section{References}

(1) de Gennes, P.-G.; Brochard-Wyart, F.; Quéré, D. Capillarity and Wetting Phenomena: Drops, Bubbles, Pearls, Waves; Springer, 2003.

(2) Landau, L. D.; Lifshitz, E. M. Fluid Mechanics; Butterworth-Heinemann, 2000.

(3) Lo, L. L. The meniscus on a needle a lesson in matching. J. Fluid Mech. 1983, 132, $65-78$.

(4) Bowden, N.; Terfort, A.; Carbeck, J.; Whitesides, G. M. Self-assembly of mesoscale objects into ordered two-dimensional arrays. Science 1997, 276, 233-235.

(5) Hosokawa, K.; Shimoyama, I.; Miura, H. Two-dimensional micro-self-assembly using the surface tension of water. Sens. Act. A 1996, 57, 117-125.

(6) Bowden, N.; Choi, I. S.; Grzybowski, B. A.; Whitesides, G. M. Mesoscale self-assembly of hexagonal plates using lateral capillary forces: synthesis using the capillary bond. $J$. Am. Chem. Soc. 1999, 121, 5373-5391.

(7) Bowden, N.; Oliver, S. R. J.; Whitesides, G. M. Mesoscale self-assembly: capillary bonds and negative menisci. J. Phys. Chem. B 2000, 104, 2714-2724.

(8) Suter, R. B. Locomotion on the water surface: Hydrodynamic constraints on rowing velocity require a gait change. J. Exp. Biol. 1999, 202, 2771-2785.

(9) Bush, J. W. M.; Hu, D. L. Walking on water: biolocomotion at the interface. Annu. Rev. Fluid Mech. 2006, 38, 339-369. 
(10) Rapacchietta, A.; Neumann, A. Force and free-energy analyses of small particles at fluid interfaces: II. Spheres. J. Colloid Interf. Sci. 1977, 59, 555 - 567.

(11) Chan, D. Y. C.; Henry, J. D.; White, L. R. The interaction of colloidal particles collected at fluid interfaces. J. Colloid Int. Sci. 1981, 79, 410-418.

(12) Vella, D.; Lee, D.-G.; Kim, H.-Y. The load supported by small floating objects. Langmuir 2006, 22, 5979-5981.

(13) Vella, D.; Mahadevan, L. The "Cheerios effect". Am. J. Phys. 2005, 817-825.

(14) Hu, D. L.; Bush, J. W. M. Meniscus-climbing insects. Nature 2005, 437, 733-736.

(15) Lumay, G.; Obara, N.; Weyer, F.; Vandewalle, N. Self-assembled magnetocapillary swimmers. Soft Matter 2013, 9, 2420-2425.

(16) Lagubeau, G.; Grosjean, G.; Darras, A.; Lumay, G.; Hubert, M.; Vandewalle, N. Statics and dynamics of magnetocapillary bonds. Phys. Rev. E 2016, 95, 053117.

(17) Nicolson, M. M. The interaction between floating particles. Proc. Cambridge Philos. Soc 1949, 45, 288-295.

(18) Kralchevsky, P. A.; Nagayama, K. Capillary interactions between particles bound to interfaces, liquid films and biomembranes. Adv. Colloid Int. Sci. 2000, 85, 145 - 192.

(19) Kralchevsky, P. A.; Nagayama, K. Particles at fluid interfaces and membranes: Attachment of colloid particles and proteins to interfaces and formation of two-dimensional arrays; Elsevier: Amsterdam, 2001.

(20) Gifford, W. A.; Scriven, L. E. On the attraction of floating particles. Chem. Engng Sci. 1971, 26, 287-297.

(21) Dixit, H. N.; Homsy, G. M. Capillary effects on floating cylindrical particles. Phys. Fluids 2012, 24, 122102. 
(22) Cooray, H.; Cicuta, P.; Vella, D. The capillary interaction between two vertical cylinders. J. Phys.: Condens. Matter 2012, 24, 284104.

(23) Vella, D.; Metcalfe, P. D.; Whittaker, R. J. Equilibrium conditions for the floating of multiple interfacial objects. J. Fluid Mech. 2006, 549, 215-224.

(24) Abkarian, M.; Protière, S.; Aristoff, J. M.; Stone, H. A. Gravity-induced encapsulation of liquids by destabilization of granular rafts. Nature Comms 2013, 4, 1895.

(25) Larmour, I. A.; Saunders, G. C.; Bell, S. E. J. Sheets of large superhydrophobic metal particles self assembled on water by the Cheerios effect. Angew. Chemie Int. Ed. 2008, $47,5043-5045$.

(26) Lu, Y.; Song, J.; Liu, X.; Xu, W.; Sun, J.; Xing, Y. Loading capacity of a self-assembled superhydrophobic boat array fabricated via electrochemical method. Micro Nano Lett. 2012, 7, 786-789.

(27) Extrand, C.; Moon, S. Using the flotation of a single sphere to measure and model capillary forces. Langmuir 2009, 25, 6239-6244.

(28) Liu, X.; Wang, X.; Liang, Y.; Zhou, F. Floating behavior of hydrophobic glass spheres. J. Colloid Int. Sci. 2009, 336, 743-749.

(29) Vella, D. Floating Versus Sinking. Annu. Rev. Fluid Mech. 2015, 47, 115-135.

(30) Mansfield, E. H.; Sepangi, H. R.; Eastwood, E. A. Equilibrium and mutual attraction or repulsion of objects supported by surface tension. Phil. Trans. R. Soc. A 1997, 869-919.

(31) Keller, J. B. Surface tension force on a partly submerged body. Phys. Fluids 1998, 10, 3009-3010.

(32) Singh, P.; Hesla, T. The interfacial torque on a partially submerged sphere. J. Colloid Int. Sci. 2004, 280, 542-543. 
(33) Pozrikidis, C. Computation of three-dimensional hydrostatic menisci. IMA J. App. Math. 2010, 75, 418-438.

(34) Abramowitz, M.; Stegun, I. A. Handbook of Mathematical Functions with Formulas, Graphs, and Mathematical Tables; Dover: New York, 1964.

(35) Liszka, T. J.; Duarte, C. A. M.; Tworzydlo, W. W. hp-Meshless cloud method. Comp. Meth. Appl. Mech. Engng 1996, 139, 263 - 288.

(36) Cooray, P. L. M. H. The Capillary Interaction Between Objects at Liquid Interfaces. Ph.D. thesis, University of Cambridge, UK, 2013.

(37) Jones, S. G.; Abbasi, N.; Ahuja, A.; Truong, V.; Tsai, S. S. H. Floating and sinking of self-assembled spheres on liquid-liquid interfaces: Rafts versus stacks. Phys. Fluids 2015, 27, 072102.

(38) Lee, D.-G.; Cicuta, P.; Vella, D. Self-assembly of repulsive interfacial particles via collective sinking. Soft Matter 2017, 13, 212-221.

(39) Berhanu, M.; Kudrolli, A. Heterogeneous structure of granular aggregates with capillary interactions. Phys. Rev. Lett. 2010, 105, 98002.

(40) Raux, P. S.; Cockenpot, H.; Ramaioli, M.; Quéré, D.; Clanet, C. Wicking in a Powder. Langmuir 2013, 29, 36363644.

(41) Ban, S.; Wolfram, E.; Rohrsetzer, S. The condition of starting of liquid imbibition in powders. Colloid. Surface. 1987, 22, 291-300.

(42) Senbil, N.; He, W.; Démery, V.; Dinsmore, A. D. Effect of interface shape on advancing and receding fluid-contact angles around spherical particles. Soft Matter 2015, 11, 4999-5003. 
Graphical TOC Entry

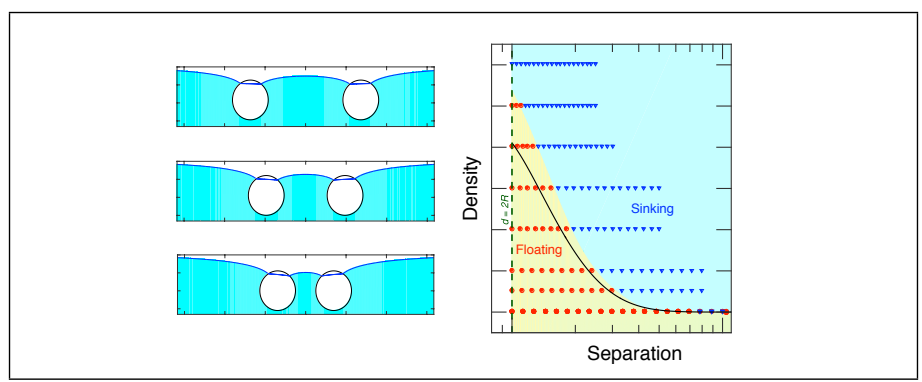

